\title{
Accesibilidad universal en el currículum universitario: un desafío necesario
}

\author{
Universal accessibility in the university curriculum: a necessary \\ challenge
}

\section{Palabras clave}

Accesibilidad universal, diseño para todas las personas, educación inclusiva, responsabilidad social universitaria, diversidad funcional, competencias curriculares.

\section{Keywords}

Universal accessibility, design for all, inclusive education, university social responsibility, functional diversity, curricular competences.

\section{Introducción}

A lo largo de la historia, las instituciones de educación superior han experimentado cambios significativos incitados por las cambiantes necesidades de la sociedad. Sin embargo, ha sido en las últimas décadas cuando las universidades han reconsiderado su rol en la sociedad (Observatorio Universidad y Discapacidad, 2012).

Siguiendo el manual la responsabilidad social universitaria y discapacidad (RSU-D), destacamos cómo nos encontramos ante una nueva realidad en la que las universidades deben desarrollar, no solamente sus funciones tradicionales de docencia, investigación y transferencia de conocimiento, sino también ser socialmente responsables en el desarrollo de sus actividades.

Las personas y la sociedad son dos realidades inseparables. La conducta humana debe medirse desde una perspectiva personal, interpersonal y social, indagando tanto en las relaciones con otras personas como en la influencia de factores sociales, por tanto, para comprender las relaciones y el comportamiento humano no podemos prescindir de las diferencias entre las personas y el medio social. Está claro que existe una necesaria comple-

\author{
María Dolores Muñoz de \\ Dios \\ <mdmunoz@ujaen.es>
}

Universidad de Jaén. España

\section{Yolanda María de la Fuente \\ Robles \\ <ymfuente@ujaen.es> \\ Universidad de Jaén. España}

\section{María del Carmen Martín \\ Cano}

<mmcano@ujaen.es>

Universidad de Jaén. España

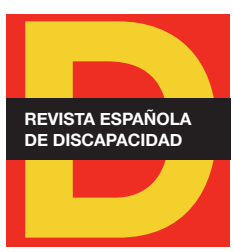

Para citar:

Muñoz de Dios, M. D. et al. (2020).

"Accesibilidad universal en el currículum universitario: un desafío necesario". Revista Española de Discapacidad, 8(I), pp. 247-259.

Doi: <https://doi.org/10.5569/23405104.08.01.14> 
mentariedad de los diferentes niveles de análisis que debemos adoptar a la hora de comprender y explicar la acción social (Álvaro, 2003). La facilitación del bienestar de las personas es un elemento clave para avanzar hacia un modelo de desarrollo sostenible, tanto por las características del diseño como por los comportamientos adecuados individuales o sociales que induce o dificulta (Imserso et al., 2006). En este sentido, la diversidad humana y sociocultural es un elemento que debe incluirse en los contenidos impartibles en el campo de la accesibilidad universal y el diseño para todas las personas (D4ALL).

La diversidad humana a la que se suman componentes como diversidad en la evolución humana, diversidad biológica, diversidad sociocultural o diversidad psicológica, hay que añadir contenidos como: relaciones funcionales persona-objeto-entorno; orientaciones sobre posibles ámbitos de estudios iniciales; interacción entre contexto y usuarios/as; relaciones funcionales persona-entorno-comunicativo; entornos virtuales; análisis de necesidades de participación y código deontológico y diseño para todos/as (Imserso et al., 2006).

De ahí el interés de mostrar un desafío necesario motivado por tres cuestiones fundamentales:

- La formación es la clave de un futuro sin exclusiones.

- El diseño para todas las personas es la base de los proyectos con la actividad humana.

- La accesibilidad universal es generadora de nuevas oportunidades para el desarrollo profesional.

Finalmente, marcamos el punto de partida de la incorporación del diseño universal en el currículum universitario, en la lucha por la formación en igualdad de oportunidades donde el avance en la aplicabilidad del diseño para todas las personas, pone de manifiesto cómo antes se dirigía a profesiones relacionados con los entornos mientras que ahora el horizonte es mucho más abierto y se enfoca a todas aquellas profesiones que prestan un servicio.

\section{Un reto al alcance de la Universidad}

La defensa hacia la Responsabilidad Social Corporativa (RSC) desde la universidad se centra en los criterios básicos que permitan adquirir competencias en accesibilidad y el desarrollo de la profesión con garantías sociales. Se trata de impulsar la generación de contenidos más justos que contribuyan a la mejora de calidad de vida.

A lo largo del tiempo conforme ha ido evolucionando el concepto responsabilidad social corporativa (RSC), ha sido analizado desde diferentes perspectivas o enfoques. En un principio, autores como Lewitt o Friedman consideraban que el deber de las corporaciones era obtener los máximos beneficios respetando la ley y unos mínimos éticos, es decir, la realización de actividades sociales, sólo se entiende para conseguir el máximo beneficio empresarial (Muñoz et al., 2015). Otro enfoque representado por autores como Mcguire, Andrews, Carroll o Epstein, consideran que las corporaciones tienen obligaciones más amplias hacia la sociedad. 
Cuando se habla de responsabilidades más amplias, nos referimos a que el concepto implica una obligación por parte de la organización, reconociendo la necesidad de la corporación de interactuar con todos los grupos que tienen algún interés en las actividades de la organización (Moreno y Capriotti, 2006).

Aunque no existe una definición consensuada sobre el término de RSC, podría ser entendida como:

\begin{abstract}
"Una estrategia de gerencia, que debe ir más allá del mero hecho del cumplimiento de la legalidad, estableciendo un marco de conocimiento en pro del beneficio social, medioambiental, ético y económico, manteniendo un equilibrio entre las cuatro dimensiones, favoreciendo e impulsando proyectos de mejora social basados en un comportamiento voluntario y proactivo" (Mármol y Medina, 2014: 28).
\end{abstract}

La incorporación de la dimensión de la discapacidad en la RSC comienza por reconocer al colectivo como un grupo de interés y desde una perspectiva amplia: no solo como empleados/as, sino también como clientes/as, proveedores, accionistas, etc. De esta forma, la RSC-D se define como "la incorporación de manera transversal de la dimensión de la discapacidad en las diferentes vertientes de la política de RSE de las empresas, considerando al colectivo de personas con discapacidad entre sus grupos de interés" (Mármol y Medina, 2014: 42).

"El compromiso social de las universidades debe verse reflejado en conductas que tengan en consideración la vida en sociedad, abogando por la dignidad de la persona, libertad, democracia, participación y solidaridad para la convivencia, equidad social y la aceptación y aprecio a la diversidad". (Muñoz et al., 2015: 4).

\title{
3. Accesibilidad como compromiso social
}

"La aspiración hacia una mayor accesibilidad ha llevado a consolidar la idea de diseño para todos o diseño universal" (Toboso-Martín y Rogero-García, 2012: 164). La European Commission (2001) y European Institut for Design and Disability (2004), señalan cómo el diseño para todos, aspira a tener en cuenta en la propia fase de diseño, los requisitos de accesibilidad derivados de los distintos tipos y grados de capacidad funcional de las personas, como objetivo al que de no tener en cuenta provoca discriminación, exclusión y problemas de participación social.

El artículo 3.5 del Real Decreto 1303/2007 de ordenación de las enseñanzas universitarias oficiales, destaca la idea de promover la incorporación de competencias transversales en accesibilidad universal y principios del diseño para todas las personas:

"Entre los principios generales que deberán inspirar el diseño de nuevos títulos, los planes de estudios deberán tener en cuenta que cualquier actividad profesional debe realizarse: b) Desde el respeto y promoción de los derechos humanos y los principios de accesibilidad universal y diseño para todos, debiendo incluirse, en los planes de estudios en que proceda". (España, 2007: 4).

A lo largo de la historia, las instituciones de educación superior han experimentado cambios significativos incitados por las cambiantes necesidades de la sociedad. 
La necesidad de poder dar respuesta a las nuevas demandas sociales es lo que motiva la defensa de incluir la accesibilidad universal y el D4ALL como competencias transversales de la formación, capaces de mejorar la excelencia y calidad adaptándose a la nueva realidad donde garantizar la diversidad y en definitiva el desarrollo social. Reclamamos una universidad que vaya más allá de sus funciones tradicionales como la docencia, investigación y transferencia de conocimiento y que apueste por el compromiso y responsabilidad social. Siguiendo la guía de responsabilidad social universitaria y discapacidad (RSU-D), entendemos que una universidad socialmente responsable es aquella que mediante su actividad en todos sus ámbitos y funciones ofrece servicios públicos que contribuyen al bienestar de la sociedad y su nivel cultural, abogando por la equidad, y reduciendo la brecha existente entre la investigación universitaria y las necesidades reales. El compromiso social de las universidades debe verse reflejado en conductas que tengan en consideración la vida en sociedad, abogando por la dignidad de la persona, libertad, democracia y participación, sociabilizada y solidaridad para la convivencia, equidad social y la aceptación y aprecio a la diversidad (Domínguez, 2009).

Introducir la formación en accesibilidad universal y D4ALL en los currículos universitarios no es una tarea sencilla, no sólo por las dificultades políticas y legales, sino también por las dificultades técnicas que comporta. "La accesibilidad universal no constituye hoy día una disciplina propiamente dicha, de la que exista un currículum establecido y una tradición pedagógica”. (Hernández-Galán et al., 2014, 125).

\title{
4. Diseño para todas las personas: aclarando conceptos
}

\begin{abstract}
La accesibilidad universal se presenta como una condición ineludible para el ejercicio de los derechos -en igualdad de oportunidades - por todos los individuos, y para, en último término, conseguir el logro de la igualdad dignidad humana, del libre desarrollo de la personalidad, de todas las personas. En ese sentido, no puede ser vista como una cuestión sujeta a la voluntad política facultativa y graciable, o como una técnica para la rehabilitación de ciertas personas. (Instituto de Derechos Humanos Bartolomé de las Casas, 2005: 57)
\end{abstract}

El diseño para todos tiene como objetivo hacer posible que todas las personas dispongan de igualdad de oportunidades y de participación en cada aspecto de la sociedad. Para conseguir esto, el entorno construido, los objetos cotidianos, los servicios, la cultura y la información, es decir, todo lo que está diseñado o hecho por personas para ser utilizado por personas, "ha de ser accesible y útil para todos los miembros de la sociedad y consecuente con la continua evolución de la diversidad humana" (Instituto Europeo de Diseño y Discapacidad, 1995: 2).

Unas de las conclusiones recogidas en relación al diseño universal provienen del Consejo de Europa sobre la introducción de principios del diseño universal en los programas de formación de las profesiones dedicadas al entorno construido (Resolución ResAP, 2001: 1), en la que se indica que el diseño universal es una estrategia encaminada a lograr que la concepción y la estructura de los diferentes entornos, productos, tecnologías y servicios de información y comunicación sean accesibles, comprensibles y fáciles de utilizar para todos del modo más generalizado, independientemente y natural posible, preferentemente sin recurrir a adaptaciones o soluciones especializadas. 
Al tratarse de una metodología de diseño que se adapta al momento, el diseño universal contribuye la prevención y la eliminación de obstáculos para la integración, tanto psicológica como educativa o con respecto al ámbito familiar, cultural, social o profesional (Soren, 2010).

Como ya ha quedado de manifiesto, tanto la accesibilidad como el D4ALL no son algo exclusivo de las personas con discapacidad, ya que todos/as en algún momento de nuestra vida tenemos limitaciones de nuestras propias capacidades funcionales.

Existe otro tipo de términos como el diseño centrado en el usuario (DCU), con el que se manifiesta la importancia de tener en cuenta a la persona y que se utiliza para describir el diseño en el que el usuario influye en el resultado final como es el diseño. Este persigue obtener información sobre usuarios/as y utilizarla para orientar el diseño y desarrollo de productos, por lo que puede mejorar la utilidad y la usabilidad de los servicios y productos (Mor, 2011). En cuanto al proceso, el diseño centrado en el usuario involucra al/a usuario/a en todas las fases en las que se desarrolla un producto, por lo que tiene el objetivo, de que, en la creación de productos, las personas que los utilicen los encuentren útiles y usables, es decir, satisfaga sus necesidades teniendo en cuenta sus características (Garreta y Mor, 2010), y para ello habrá que adentrarse en criterios de diseño para todos.

\section{Universidad como garantía de derechos}

A través de la Ley $51 / 2003$, de 2 de diciembre, de igualdad de oportunidades, no discriminación y accesibilidad universal de las personas con discapacidad y el Real Decreto Legislativo 1/2013, de 29 de noviembre, por el que se aprueba el Texto Refundido de la Ley General de derechos de las personas con discapacidad y de su inclusión social, se pone de manifiesto:

"La necesidad de incluir la formación en diseño para todas las personas en las enseñanzas universitarias, estableciendo la existencia de una sensibilización creciente y necesaria respecto a la igualdad de oportunidades, con la finalidad de que las instituciones universitarias formen a profesionales capaces de construir una sociedad cada vez más inclusiva" (Muñoz y Cortés, 2015: 211).

Así mismo y dentro del marco legal, es fundamental reflejar la Convención sobre los derechos de las personas con discapacidad, como instrumento destinado a proteger los derechos de las personas con discapacidad, reafirmando el artículo 4 Obligaciones generales, la importancia de promover la formación de los profesionales y el personal que trabaja con personas con discapacidad a fin de prestar mejor asistencia y los servicios garantizados en los derechos (Convención sobre los derechos de las personas con discapacidad, 2006).

A nivel europeo destacamos la Estrategia Europea sobre Discapacidad 2010-2020, que pretende poner de manifiesto el compromiso renovado para una Europa sin Barreras. Esta estrategia recoge como ámbitos de actuación la accesibilidad, participación, igualdad, empleo, educación y formación, protección social, 
sanidad, acción exterior y accesibilidad. Por la relación que guarda con nuestro tema, destacamos la accesibilidad, entendida como una condición previa a la participación en la sociedad y pretende garantizar la accesibilidad a los bienes y servicios, en especial los servicios públicos y los dispositivos de apoyo para las personas con discapacidad (Comisión Europea, 2010). Otro de los ámbitos de actuación que destacamos, es la educación y formación, donde se expresan los esfuerzos encaminados, por un lado a suprimir las barreras jurídicas y organizativas que se presentan a las personas con discapacidad en los sistemas generales de educación y aprendizaje permanente, y también a apoyar oportunamente una educación inclusiva, un aprendizaje personalizado y una identificación temprana de necesidades especiales así como facilitar una formación y un apoyo adecuado a los profesionales que trabajan en todos los niveles educativos (Diario Oficial de la Unión Europea, 2012).

El Real Decreto 1393/2007, de 29 de octubre, por el que se establece la ordenación de las enseñanzas universitarias oficiales, contempla la incorporación de materias sobre accesibilidad universal y diseño para todos en los currículos formativos, por lo que la inclusión en este nuevo paradigma, presenta la oportunidad de adaptarse a las nuevas demandas de una manera uniforme e integral en todas las profesiones de manera conjunta. A su vez, tiene que ser considerado como una necesidad de innovación metodológica para la profesión, la docencia y la investigación en lo relativo a la inclusión de las competencias de accesibilidad universal y diseño para todos, dando respuesta a una nueva manera de hacer.

\section{Metodología}

Para la consecución de los objetivos propuestos y el cumplimiento del propósito de este artículo, se realiza un análisis que engloba aspectos sobre la necesidad de incluir la accesibilidad universal y el diseño para todas las personas en los currículos formativos, configurándose como un desafío necesario en la sociedad de nuestros días.

Se abordan los conceptos de accesibilidad universal, diseño para todas las personas y diseño centrado en el usuario. Para ello, se ha realizado una revisión bibliográfica que pone de manifiesto la situación actual en relación al marco legal y el horizonte abierto sobre la discapacidad, a través de las fundamentaciones de la legislación española o la Convención de Naciones Unidas sobre los Derechos de las Personas con Discapacidad.

De manera paralela, señalamos las buenas prácticas detectadas en la oferta académica en relación a la adquisición de competencias en materia de accesibilidad universal y diseño para todas las personas, donde el fin es reivindicar que nos encontramos ante una oportunidad de cambio para la cual la universidad puede dar respuesta. 


\section{Resultados}

Con el objetivo puesto en que las universidades españolas impulsen un programa de accesibilidad universal en el currículum formativo universitario, y como resultado de la implicación por incluir en los planes de estudios el D4ALL, como ejemplos de buenas prácticas, se encuentra en primer lugar la iniciativa de la Coordinadora del Diseño para Todas las Personas en España. Esta entidad en colaboración con el Imserso, editó en 2006, el Libro blanco de diseño para todos en la universidad, lo que dio lugar en 2010 a la realización de diversas publicaciones sobre formación curricular en diseño para todos en las disciplinas más relacionadas con el entorno construido y las tecnologías de la información y la comunicación. Estas publicaciones ponen de manifiesto el interés de las universidades públicas españolas en ser realmente inclusivas y en proporcionar una formación necesaria que la sociedad demanda y requiere, para el uso y disfrute de los servicios en relación a todos los ámbitos de la vida en la ciudad (CRUE, 2014).

Existen doce manuales que contemplan la implementación de los principios de igualdad de oportunidades y accesibilidad universal en titulaciones de grado. Actualmente se está trabajando en los manuales relacionados con las titulaciones de Turismo y Educación, como Educación Infantil, Educación Primaria, Educación Social y Máster en Profesorado.

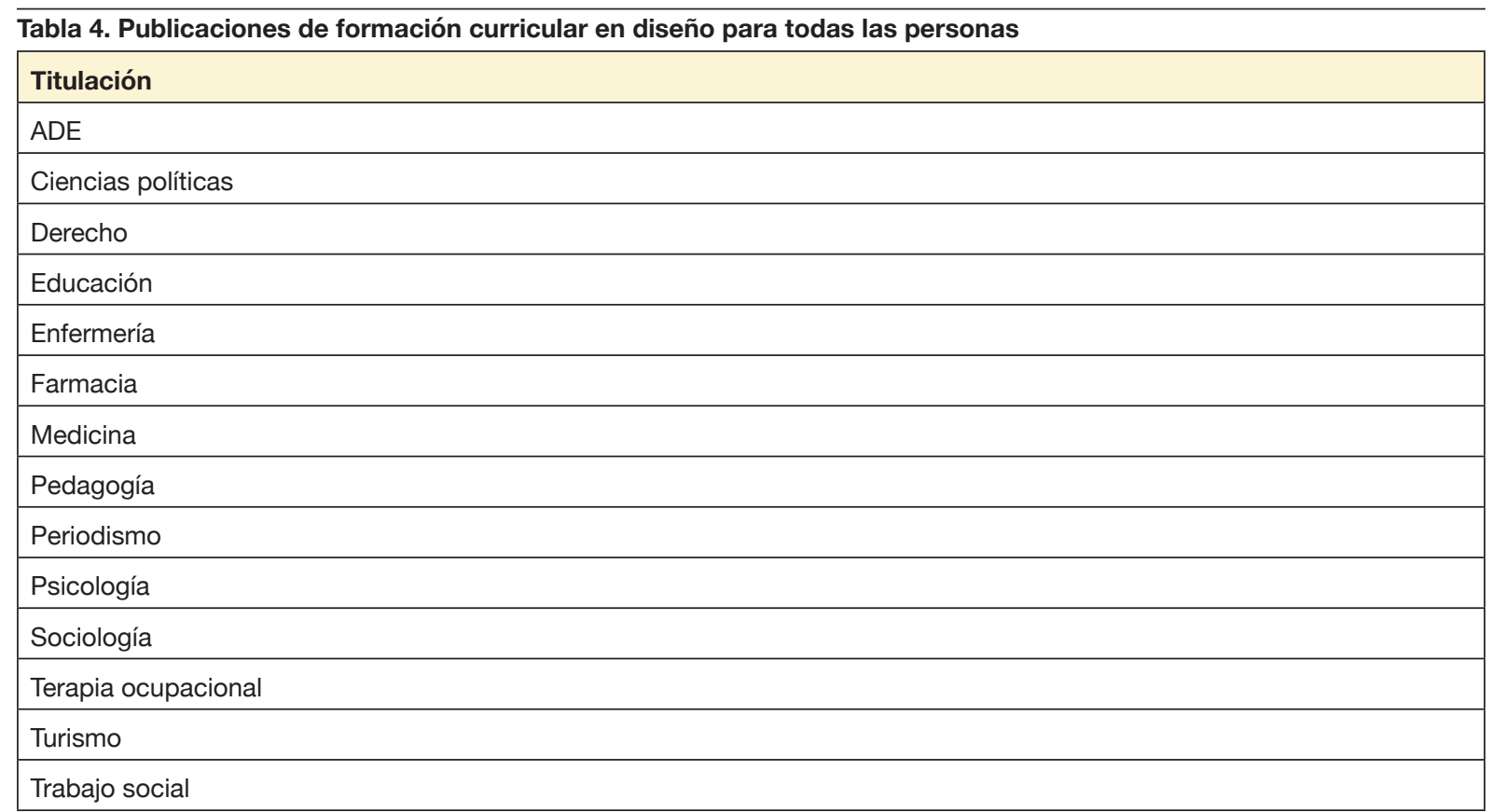

Fuente: elaboración propia a partir de la Web www.crue.org.

Otro ejemplo de buenas prácticas que encontramos en relación a la adquisición de competencias en accesibilidad para hacer realidad un ejercicio profesional inclusivo, es el curso en abierto desarrollado en la 
Universidad de Jaén en colaboración con el Real Patronato sobre Discapacidad y Fundación ONCE. Se trata de un curso con carácter general e innovador, abierto a todas aquellas personas interesadas en la accesibilidad universal y el diseño para todas las personas, cuya realización es bajo la modalidad online 2.0, donde se da la oportunidad tanto al alumnado como egresados y profesionales a que sean la herramienta para que muchas personas desarrollen su proyecto de vida en igualdad, haciéndoles conseguir la conquista de lo cotidiano.

\begin{tabular}{l}
\hline Tabla 5. Estructura de contenidos. Curso en abierto: diseño accesible, diseño para todas las personas \\
\begin{tabular}{|l|}
\hline Introducción a la accesibilidad universal \\
\hline Certificación en accesibilidad universal \\
\hline Transversalidad y discapacidad \\
\hline Fundamentos de usabilidad \\
\hline Diversidad como factor de inclusión \\
\hline Interacción persona entorno \\
\hline Tercer sector y accesibilidad \\
\hline Accesibilidad universal en Latinoamérica y buenas prácticas en: España, Ecuador, Colombia, Bolivia, Perú y Chile \\
\hline
\end{tabular}
\end{tabular}

Fuente: elaboración propia a partir de la web www.ujaen.es.

Ponemos de nuevo en evidencia la trayectoria del Máster Propio en "Accesibilidad Universal y Diseño para Todos" realizado desde la Universidad de Jaén en colaboración con Fundación ONCE y que comenzó en el año 2011 con un carácter presencial, donde seis años más tarde, continúa formando a profesionales de diferentes ramas profesionales. Desde el año 2014, el máster modificó su modalidad presencial y se configuró como el I Máster en Accesibilidad para Smart City. La ciudad global, donde con un carácter online cubre las necesidades de formación en accesibilidad destinadas a todos los servicios de la ciudad.

Tabla 6. Estructura de contenidos. Máster propio en Accesibilidad para Smart City. La Ciudad Global

\begin{tabular}{|l|l|}
\hline Modulo & Asignatura \\
\hline Módulo común 1. La ciudad & - La ciudad. Evolución de la ciudad. Tipos de ciudad \\
& - La ciudad global \\
& - Smart City A+ \\
& - Los servicios de la ciudad \\
\hline \multirow{4}{*}{ Módulo común 2. La ciudadanía } & - Diversidad ciudadana \\
& - Igualdad de oportunidades \\
& - Marco legal \\
& - El diseño de la ciudad centrado en la persona \\
\hline \multirow{3}{*}{ Módulo optativo. Los servicios de la ciudad } & - Movilidad y transporte \\
& - Urbanismo \\
& - Vivienda \\
& - Disciplina urbanística \\
& - Accesibilidad en edificios de uso publico \\
& - Educación - Cultura \\
& - Servicios sociales y salud \\
& - Turismo \\
\hline
\end{tabular}




\begin{tabular}{|l|l|}
\hline Modulo & Asignatura \\
\hline \multirow{3}{*}{ Módulo optativo. Los servicios de la ciudad } & - Deporte y ocio \\
& - Accesibilidad universal en la sociedad de la información \\
& - Diseño para todos en hardware, software y web \\
& - Deño para todos en medios y terminales públicos \\
& - Organización y desarrollo de la accesibilidad universal \\
\hline Talleres de innovación & - Protocolo accesible \\
& - Accesibilidad low cost \\
& - Musicoterapia \\
\hline
\end{tabular}

Fuente: elaboración propia a partir de la web www.masteraccesibilidad.ujaen.es

\section{Discusión y conclusiones}

Según lo expuesto, nos encontramos actualmente ante una oportunidad de cambio motivado por el avance positivo que está experimentando la accesibilidad universal y el D4ALL. Hasta ahora se entendía como un paradigma aplicable a los/as profesionales que diseñaban los entornos en los que nos desenvolvemos día a día, pero dando un paso más, pretende incorporarse en aquellos/as que prestan un servicio. Tanto los/as profesionales que ejercen su profesión como aquellos/as que están en fase de formación, necesitan competencias específicas en diseño para todas las personas. De esta forma se podrá hacer de su desarrollo profesional una oportunidad y no una nueva barrera, obteniendo una sociedad justa (Muñoz et al., 2015).

Los estudios empíricos sobre responsabilidad social en el ámbito educativo se han quintuplicado en la última década, donde las universidades son el foco de interés (Gordon y Gelardi, 2005). Una de las dimensiones que se encuentra dentro de la responsabilidad social universitaria es la accesibilidad social de la universidad, que pretende brindar a sus estudiantes oportunidades de interacción con diversos sectores sociales poniendo de manifiesto una vinculación con la sociedad (Vallaeys, 2008). Apoyados en Moriña-Diez et al. (2013), ponemos en evidencia cómo la falta de formación del profesorado para atender al alumnado con discapacidad provoca en ciertos casos que sean los propios estudiantes los que informen de las necesidades para poder seguir el desarrollo de la docencia. Así mismo existen denuncias en cuanto a la falta de formación para atender discapacidades, ya que esto afecta no sólo al profesorado, sino a los propios estudiantes siendo necesario recibir más formación sobre las adaptaciones y necesidades, lo que ocasiona la demanda sobre la creación de programas y/o asignaturas, cuyo objetivo sea dotar tanto al alumnado como al profesorado la formación necesaria para atender de forma adecuada al colectivo de personas con discapacidad. No obstante, observando el panorama europeo sobre la población de estudiantes universitarios con discapacidad y comparándolo con las universidades españolas, hay grandes diferencias que se centran en el progreso de la universidad española en la última década (Castellana y Sala, 2005). Aun así, sigue siendo necesario avanzar más para que los derechos de las personas con discapacidad sean seguros, pudiendo lograr que la universidad española sea tanto competente como compatible con respecto a las del resto de Europa (Mirón et al., 2005). 
A favor de la legislación y en pro de afrontar las necesidades de las personas con discapacidad, la universidad debe integrar en la realidad actual del sistema educativo puntos como: edificios, instalaciones, dependencias y espacios virtuales; igualdad de oportunidades de los/as estudiantes; atención integral de las personas con discapacidad o adaptaciones curriculares. De este modo garantizaría la cadena de accesibilidad en la universidad y con ello, satisfacer las necesidades originarias del sistema educativo (Fundación ONCE, 2010).

Motivados en la defensa y apoyo a los derechos humanos y a la justicia social, y principalmente, en no trabajar para las personas sino con ellas, el nuevo enfoque destinado a la accesibilidad universal en el $\mathrm{cu}$ rrículum universitario, hace que sea necesario adquirir competencias específicas para hacer del desarrollo profesional una oportunidad y no una nueva barrera, lo que supone una oportunidad de cambio para la mejora de la accesibilidad de la ciudadanía ya que consigue:

- Aumento de la cohesión social, de la igualdad y el equilibrio territorial y económico.

- Contribuye a la mejora en las prestaciones.

- Favorece la puesta en marcha de nuevas actividades laborales, económicas, culturales y sociales.

La universidad se constituye como un microcosmos de la sociedad de la que forma parte, por ello debe incorporar la igualdad de oportunidades y la accesibilidad universal y el diseño para todos. Debe ser útil y contribuir a su desarrollo, y en el sector de la discapacidad, todavía resta mucho por hacer para logar la plena integración social de las personas con discapacidad (Briceño, 1989). Desde esta perspectiva, defendemos la discapacidad como tema de investigación, siendo una función principal de las universidades, tal y como lo refleja la Ley Orgánica de Universidades 4/2007 declarando "la investigación científica como fundamento esencial de la docencia y herramienta primordial para el desarrollo social a través de la transferencia de sus resultados a la sociedad" (Fundación Universia, 2016: 54), por lo que la inclusión de la diversidad en general y de la discapacidad en particular contribuyen a la consecución de una sociedad más justa e inclusiva.

No podemos finalizar sin destacar la importancia en la concienciación de los/as estudiantes en la creación de entornos respetuosos con la diversidad humana, así como de la necesidad porque la accesibilidad universal se incorpore en todos los planes de estudios. El propósito es mostrar y justificar la necesidad de su implantación donde las universidades garanticen el respeto de los derechos humanos y la visión de la diversidad (Cano et al.,2013). 


\section{Referencias bibliográficas}

Álvaro, J. L. (2003). Fundamentos sociales del comportamiento humano. Barcelona: Editorial UOC.

Briceño, R. (1989). La universidad como un microcosmos de conflicto social. La política de reforma de la universidad nacional de Colombia 1964-1974. Conferencia anual de la Asociación Americana de Investigación en Educación AERA. Universidad de Stanford, Estados Unidos. Recuperado de: http://www.bdigital.unal.edu. co/41152/1/12198-31059-1-PB.pdf.

Cano, N. et al. (2013). Nuevos retos de futuro para la universidad: la integración de asignaturas sobre discapacidad y accesibilidad universal en la formación curricular de las titulaciones actuales. Universidad de Alicante. Recuperado de: https://rua.ua.es/dspace/bitstream/10045/44276/1/2013-XI-Jornadas-Redes-192.pdf.

Castellana, M. y Sala, I. (2006). "La inclusión de los estudiantes con discapacidad en la universidad: un reto para la universidad española en el nuevo espacio europeo de la educación superior”. Revista de psicología 18, pp. 209-227.

Comisión Europea (2010). Estrategia Europea sobre Discapacidad 2010-2020: Un compromiso renovado para una Europa sin barreras. Bruselas: Comisión Europea.

Comité de Ministros del Consejo de Europa (2001). Resolución ResAP sobre la introducción de los principios de diseño universal en los planes de estudio de todas las actividades profesionales relacionadas con el entorno de la construcción. Diario Oficial de las Comunidades Europea, 15 de febrero de 200.

CRUE (2014). Formación Curricular en diseño para todas las personas. Recuperado de: http://www.crue.org/SitePages/Formacion-Curricular-Diseno-para-todas-las-personas.aspx.

Diario Oficial de la Unión Europea. (2012). Conclusiones del Consejo de 12 de mayo de 2009. Recuperado de: https://op.europa.eu/en/publication-detail/-/publication/f349e9ff-9cb8-4f73-b2f6-0a13452d22b4/languagees/format-PDF.

Domínguez, M. J. (2009). Responsabilidad social universitaria. Humanismo y trabajo social, (8), pp. 37-67.

España. Decreto 1393/2007, de 29 de octubre, por el que se establece la ordenación de las enseñanzas universitarias oficiales. Boletín Oficial del Estado, 30 de octubre de 2007, núm. 260.

España. Ley 51/2003, de 2 de diciembre de igualdad de oportunidades, no discriminación y accesibilidad universal de las personas con discapacidad. Boletín Oficial del Estado, 3 de diciembre de 2003, núm. 289.

España. Real Decreto 1/ 2013, de 29 de noviembre, por el que sea prueba el Texto Refundido de la Ley General de derechos de las personas con discapacidad de su inclusión social. Boletín Oficial del Estado, 3 de diciembre de 2013, núm. 289.

European Commission. (2001). Background Paper "Discrimination by Design Conference. Bruselas: European Commission.

European Institut for Design and Disability (2004). Declaración de Estocolmo. Recuperado de http://www.designforalleurope.org/upload/design\%20for\%20all/sthlm\%20declaration/stockholm\%20decla-\%20ration_spanish.pdf.

Fundación ONCE (2010). La accesibilidad del entorno universitario y su percepción por parte de los estudiantes con discapacidad. Observatorio Universidad y Discapacidad. Universidad Politécnica de Cataluña. Recuperado de: https://upcommons.upc.edu/bitstream/handle/2117/10243/OUD1\%202010-\%20cas.pdf. 
Fundación Universia (2016). Universidad y discapacidad. III Estudio sobre el grado de inclusión del sistema universitario español respecto de la realidad de la discapacidad. Recuperado de: http://riberdis.cedd.net/bitstream/ handle/11181/5199/Universidad_y_discapacidad_III_Estudio.pdf?sequence=1\&rd=0031788541347956.

Garreta, M., y Mor, E. (2010). Diseño centrado en el usuario. Universitat Oberta de Catalunya. Recuperado de https://www.exabyteinformatica.com/uoc/Informatica/Interaccion_persona_ordenador/Interaccion_persona_ ordenador_(Modulo_3).pdf.

Gordo, I. y Gelardi, A. (2005). "Factors that affect understanding of social responsability accounting". Canadian Accounting Perspectives, 4 (1), pp. 31-59.

Hernández-Galán, J. et al. (2014). "La accesibilidad universal y el diseño para todas las personas factor clave para la inclusión social desde el design thinking curricular”. Revista d'intervenció Socioeducativa, 58, pp. 119-134.

Instituto de Derechos Humanos Bartolomé de las Casas (2005). El significado de la accesibilidad universal y su justificación en el marco normativo español. Recuperado de https://www.cermi.es/sites/default/files/docs/colecciones/Elsignificadodelaaccesibilidaduniversalysujustific.pdf.

Instituto Europeo de Diseño y Discapacidad. (1995). La Declaración de Estocolmo del EIDD. Recuperado de http:// www.upv.es/contenidos/CAMUNISO/info/U0579218.pdf.

Imserso et al. (2006). Libro blanco del diseño para todos en la universidad. Recuperado de https://www.uab.cat/ Document/994/336/libro_blanco_universidad,0.pdf.

Mármol, D., y Medina, J. M. (2014). La accesibilidad desde la responsabilidad social corporativa. Madrid: Oportunidad Accesible.

Mirón, J. A. et al. (2005). Universidad y discapacidad: diagnóstico de situación. I Congreso nacional sobre universidad y discapacidad. Salamanca: Gráficas LOPE.

Mor, E. (2011). Diseño centrado en el usuario. Universitat Oberta de Catalunya. Recuperado de https://estudios. uoc.edu/es/masters-posgrados-especializaciones/especializacion/dise\%C3\%B1o-creacion-multimedia/diseno-usuario-investigacion/profesorado.

Moreno, Á., y Capriotti, P. (2006). "La comunicación de las empresas españolas en sus webs corporativas. Análisis de la información de responsabilidad social, ciudadanía corporativa y desarrollo sostenible”. Zer, 21, pp. 47-62.

Moriña-Diez, A. et al. (2013). "El profesorado en la universidad ante el alumnado con discapacidad: ¿tendiendo puentes o levantando muros?". Revista de docencia universitaria, 11(3), pp. 423-442.

Muñoz, M. D. y Cortés, J. (2015). Diversidad funcional: aportaciones sobre discapacidad e invalidez estructural. Formación curricular en diseño para todas las personas en trabajo social. (p. 2010-212). Revista d'intervenció sócioeducativa Núm. 58. Recuperado de http://www.raco.cat/index.php/EducacioSocial/article/viewFile/284972/372829.

Muñoz, M. D. et al. (2015). Promoción e inclusión social desde el sector turístico empresarial a través de la accesibilidad universal y el diseño para todas las personas. Congreso Seguridad Turística. Recuperado de http://congresos.seguridadturistica.org/ponencias/TURISMO_ACCESIBLE/13-MariaDoloresMunozdeDiosyotros.pdf

Naciones Unidas (2006). Convención sobre los derechos de las personas con discapacidad. Recuperado de https://www.un.org/esa/socdev/enable/documents/tccconvs.pdf.

Observatorio Universidad y Discapacidad (2012). La responsabilidad social universitaria y discapacidad (RSU-D). Recuperado de http://www.fundaciononce.es/sites/default/files/docs/Guia\%2520RSU-D\%25202012_2.pdf. 
Soren, G. (2010). Hacia la plena participación mediante el diseño universal. Madrid: Ministerio de Sanidad y Política Social.

Toboso-Martín, M. y Rogero-García. J. (2012). "Diseño para todos en la investigación social sobre personas con discapacidad”. Reis, 140, pp. 163-172. Doi: 10.5477/cis/reis.140.163.

Vallaeys, F. (2008). "Responsabilidad social universitaria: una nueva filosofía de gestión ética e inteligente para las universidades". Revista Educación Superior y Sociedad, Nueva Época. El movimiento de responsabilidad social de la universidad: una comprensión novedosa de la misión universitaria 13(2), pp. 191-220. 